\title{
Enhancing engagement in CAMHS
}

\section{Joanna Appleby and Deb Phillips}

Joanna Appleby is a Registered Social Worker. She began work at the Kari Centre (central Auckland child and adolescent mental health service) in 2007 before joining the Regional Youth Forensic Service in 2011. Joanna has a passion for working with vulnerable adolescents and addressing barriers to engagement with mental health services.

Deb Phillips is a Registered Social Worker. She has been working at the Kari Centre since 2006. Deb is particularly interested in mental and emotional wellbeing, with a focus on the importance of early intervention and promotion of healthy thinking in young people that will serve them well into their future.

\section{Abstract}

Child and adolescent mental health services (CAMHS) are tasked with working with young people with moderate-severe mental health needs. However, for many of those young people who are in most need, there are several barriers to engaging with CAMHS. These barriers can be environmental, cultural and psychological.

For CAMHS to provide effective services, these barriers must be addressed by both clinicians (including social workers) and referrers. A review of the literature suggests engagement interventions that target all levels of barriers, providing an ecological approach to enhancing engagement. New Zealand CAMHS are trying new initiatives to improve access rates. Social work values of choice, self-determination and collaboration underpin these interventions.

\section{Introduction}

There are 20 child and adolescent mental health services (CAMHS) in New Zealand (The Werry Centre, 2011). CAMHS are multidisciplinary, including social workers, psychologists, psychiatrists, child psychotherapists, occupational therapists and nurses. These teams deliver mental health assessment and intervention to young people and their families, with a target of reaching $3 \%$ of the age-specific population.

It is well documented that New Zealand youth have high rates of mental health difficulties, particularly depression, anxiety, substance use and suicide (Mental Health Commission, 2012). The Youth 2007 data showed that $15 \%$ of female students and $7 \%$ of male students reported significant symptoms of depression (Adolescent Health Research Group, 2008), and suicide rates among New Zealand youth remain high, with the second highest rate of youth (15-24 years) suicide in the OECD (Ministry of Social Development, 2010). It is clear that young people in New Zealand have a range of mental health needs.

Given that mental health difficulties are so common amongst youth, the Government has indicated a priority to address youth mental health (Ministry of Health, 2007). The recent 
release of the Blueprint II, outlining strategic plans for the development of mental health services, places a large emphasis on the importance of early intervention, particularly for youth (Mental Health Commission, 2012). There is a vision to remove wait lists and improve access to services for all New Zealanders, with equality of outcomes for all. The document refers to people-centred practice with a focus on recovery and resiliency. This sits well with social work values of empowerment and strengths-based practice.

\section{Engagement}

\section{Reaching those in need}

New Zealand youth are a unique group, comprising multiple cultures and a range of attitudes towards emotional wellbeing and help-seeking. While there is a goal of reaching $3 \%$ of the population, CAMHS are seeing only $1.49 \%$ of the population (The Werry Centre, 2011). Sometimes it is those young people who are most in need of mental health services, that are the least likely to be referred or engage with CAMHS. Treatment rates are low, and many young people with mental health needs do not connect with CAMHS (McKay \& Bannon, 2004).

In practice, there are many young people who are referred with serious mental health concerns who then minimise these issues when first seen by CAMHS. These are the young people who say ' $\mathrm{I}$ ' $\mathrm{m} \mathrm{OK}^{\prime}$ and report that they do not require any specialist intervention. While we must respect client's self-determination and autonomy, this can be understood in the context of personal and societal barriers to engagement. There are a number of young people who disclose their distress quite some time after first meeting a clinician. This process of slow engagement appears to occur more with young people who have a strong sense of self-reliance, and a mistrust of mental health services. There are also parents who do not wish CAMHS to be involved with their children initially, but after efforts are made to build rapport and trust, they can be more willing to engage.

Engaging these hard-to-reach and hard-to-engage young people and families is a challenge for CAMHS clinicians. However, it is important to make efforts to enhance engagement in order to care for our most vulnerable and to work towards a healthy future in New Zealand. Identifying barriers to engagement and addressing these through targeted interventions can increase access rates and improve the mental health of New Zealand youth.

\section{Defining engagement}

There are several different phases within the concept of engagement. McKay and Bannon (2004) identify that engagement begins with the recognition that a mental health problem exists. The next phase is the connection of the young person with a mental health service via a referral from an involved professional. The third step is the agreement that the young person will be seen by the service, followed by initial attendance. Referral to CAMHS and initial assessment are the focus of this review, examining the ways in which CAMHS clinicians and other involved professionals can enhance engagement from the first point of contact with CAMHS. The final phase is ongoing engagement.

Sometimes in practice, when young people or families do not attend appointments, this can be viewed as a fault of the family. Team discussions may be about a client's 'resistance', (un)readiness for change and lack of insight into their mental health needs. Often these ideas 
arise in the context of high workloads and staff shortages, factors that increase pressure on CAMHS clinicians. The onus of engaging is then placed on the young person and their family, rather than as a shared responsibility. This can lead to pathologising families as a result of engagement issues.

Social workers operating from a systemic view have the benefit of understanding engagement issues from a macro level. An understanding of contextual barriers to engagement shifts the responsibility of engagement more towards the clinician and other professionals involved. This review of engagement interventions is intended to provide clinicians and referrers with ideas about what we can do as professionals to enhance engagement and increase access for those most in need of mental health services.

\section{Barriers to engagement}

Before engagement issues can be addressed, the barriers to engagement must first be identified. These engagement barriers span many areas, from personal psychological barriers, to cultural beliefs, practical needs, agency limitations and societal stigma.

\section{Psychological barriers}

Understanding and expectations of mental health services can be a significant barrier to engagement. McKay and Bannon (2004) identified that parental perceptions of the service are very important - particularly their expectations, alliance with the clinician and the degree to which they perceive the needs of their child. Those parents who have low expectations of CAMHS and do not think that their child has a mental health need are less likely to engage in services. The same is true of young people. Rickwood, Deane, Wilson \& Ciarrochi (2005) identified that lack of emotional competence and negative attitudes about help-seeking are common barriers for young people to seek help for emotional issues. Young people can have a preference for self-reliance and may see help-seeking as a sign of weakness (Gulliver, Griffiths \& Christensen, 2010).

\section{Cultural barriers}

There can be many different cultural understandings of mental health. There are five Maorispecific services nationwide, and two Pacific services (The Werry Centre, 2011). However, most of the CAMHS are mainstream services, and thus there can be access barriers for people from minority cultures.

This is particularly true for Maori: Maori youth are reported to have twice the rate of mental health problems compared to non-Maori youth (Fergusson, Poulton, Horwood, Miln \& Swain-Campbell, 2003). While 3\% was deemed the appropriate general access rate for CAMHS, it was suggested that Maori access should be $6 \%$, however Maori access rates in 2009 were 1.76\% (The Werry Centre, 2011). The Youth 2007 data identified what Maori youth reported as barriers to accessing healthcare. These included being 'too scared', 'don't want to make a fuss', 'couldn't be bothered', financial limitations to attending appointments, lack of transport and difficulties getting to appointments (Clarke, et al., 2008). While access rates are improving for Maori, there still remain cultural barriers that make it difficult for Maori young people to seek help from a mainstream CAMHS. These can often be related not only to the above, but also to differing perceptions of mental health issues. 
Access rates are lower for Pacific and Asian clients. In 2009 only $0.99 \%$ of the Pacific population accessed CAMHS, while the access rate for the Asian population was $0.45 \%$ (The Werry Centre, 2011). It was hypothesised that the Asian access rate was due to the stigma of mental health and a lack of understanding of services available. Shame is also a factor that has been identified by Asian mental health professionals as a barrier to accessing mental health services. Pacific access rates are affected by cultural explanatory models of mental illness that differ from medical models, along with stigma about attending mental health services (The Werry Centre, 2011).

\section{Other barriers}

Some families are prevented from accessing CAMHS due to practical needs. These are concrete obstacles such as insufficient time, lack of transport and lack of childcare options (McKay \& Bannon, 2004). Some parents feel unable to take time off work for clinic appointments. These obstacles are more likely to exist for those who are economically disadvantaged. Poverty can be a real barrier, especially considering that low income communities have higher rates of youth with mental health issues (McKay \& Bannon, 2004).

Families report that they find it difficult to engage with CAMHS when they are placed on a waitlist (Ministry of Health, 2007). Another agency limitation can be reduced flexibility for appointment times due to high workload. Also, the expectation that parents are involved with CAMHS can be a barrier for some young people. Many young people do not like to share information with their parents and struggle with the idea of their parents being aware of their mental health needs.

On a macro level, societal stigma about mental health is a barrier that can be both psychological and cultural (Rickwood et al., 2005). Social stigma around mental health can cause embarrassment and a reluctance to seek help (Gulliver et al., 2010). It is important for clinicians and referrers to be aware of these social pressures and to address these barriers.

\section{Literature review of current interventions}

\section{Psychological interventions}

The literature on engagement interventions highlights the need to address barriers at all levels of engagement (Ingoldsby, 2010; Kim, Munson \& McKay, 2012; McKay \& Bannon, 2004). In regards to psychological barriers, it is suggested that clinicians work with the negative perceptions that young people and families may have about treatment, and that this occurs from the very first contact (Kim et al., 2012). This kind of approach involves the use of motivational interviewing, identifying the benefits of the service and talking about family expectations of CAMHS (Ingoldsby, 2010). While a young person may have been referred for anxiety, they may be more interested in using therapy to address other issues first, such as rebuilding relationships with friends. Having a collaborative approach to goal setting and treatment plans builds trust and brings the expectations of the service and the client closer.

There is a role for other professionals involved, in order to promote mental health services and encourage positive expectations of CAMHS (Rickwood et al., 2005). Given that psychological barriers about help-seeking are so common, referrers and other involved professionals have an important role in modeling positive attitudes about help-seeking and CAMHS. 


\section{Cultural interventions}

In regards to cultural barriers for Maori clients, The Werry Centre (2011) has made several recommendations to increase access for Maori clients. It is suggested that there should be more kaupapa Maori mental health services, that mainstream services should be both clinically and culturally competent, the Maori workforce increased, early intervention strategies developed and Maori clients are worked with to identify effective strategies to increase access. Given that most Maori clients attend a mainstream, rather than a kaupapa Maori service it is essential that CAMHS be both clinically and culturally competent (The Werry Centre, 2011; Willie, 2006).

One of the ways that this can occur is through the use of cultural brokers. Bryan (2009) describes these workers as people who have knowledge of both cultures and facilitate clear communication between both parties. Cultural brokers can be cultural advisors employed by CAMHS, or could be lay people from the community who are comfortable in both cultures (for example a person familiar with mainstream CAMHS and also understanding of another cultural view of mental health).

Compulsory and regular staff training is also important in order to keep non-Maori staff aware of current issues and developments in Maori thought regarding mental health issues, barriers to accessing services and protocol for engaging with Maori. This is particularly important for social workers with a commitment to the Treaty of Waitangi and Maori as tangata whenua. At a service level, regular training becomes even more important so that clinicians do not rely solely on cultural brokers to facilitate engagement.

There is a lack of relevant research about increasing Pacific and Asian access to CAMHS, as much of the research has focused on enhancing Maori access. However, the research about cultural brokers and cultural competency can be applied to all cultural groups.

\section{Appointment reminders}

There is little information in the literature about how to address practical needs that are barriers to engagement. The main intervention that has been researched is reminding clients about appointments, either through letters, phone calls or text reminders. In a study of general health appointments, attendance at the clinic increased by $50 \%$ for those who received text reminders (Guy, Hocking, Wand, Stott, Ali \& Kaldor, 2012). McKay and Bannon (2004) identified that phone reminders for CAMHS appointments improved attendance by $32 \%$, however they also acknowledged that some people have no phone and/or have frequent changes of address that limit this intervention. Unfortunately, it can be those who are most difficult to engage who do not have regular contact information.

\section{Family interventions}

Community and social support are important factors for engagement. Pescosolido, Brooks Gardner and Lubell (1998) identified that social networks can be a significant motivating factor for adults to enter mental health services. They identified that it was often the concerns from trusted others that precipitated the engagement process. Encouragement from others also enhances engagement in CAMHS (Gulliver et al., 2010). It is often parents and teachers whose concern about a young person leads to a referral to CAMHS. Working with the social structure, particularly the family, is seen as critical to enhancing engagement. Working from a viewpoint of seeing the family as partners in care can be very effective (Kim et al., 2012). 


\section{Practice initiatives}

CAMHS in New Zealand have implemented several strategies to enhance engagement, at national and service levels, and from ideas that have come from resourceful and passionate clinicians.

\section{Choice and Partnership Approach}

The Choice and Partnership Approach (CAPA) is a system designed for CAMHS to manage capacity and demand (York \& Kingsbury, 2009). It began in the UK and is now operating in New Zealand and parts of Australia (Mental Health Foundation, 2009). Of the 20 CAMHS in New Zealand, 16 are using CAPA, or at least some aspects of it. The aim of CAPA is to make CAMHS more accessible and to reduce waiting lists (Mental Health Foundation, 2009).

CAPA operates on the idea that all referred families are offered a 'choice appointment'. This is a face-to-face appointment to understand what the family needs and to reach a shared understanding of the issues. The family is offered a choice of day, time, venue, clinician and intervention, with the appointment taking place within seven days of the referral. The intention is that this choice appointment will have a conversational style to increase engagement. If the client and the clinician agree that there should be further appointments, they move to partnership appointments. In doing this, the family enters into a therapeutic contract with the service, thereby increasing buy-in for the family (Mental Health Foundation, 2009).

Research from the implementation of CAPA in the UK indicates that the system is effectively reducing waitlists (Robotham, James \& Cyhlarova, 2010). However, the research has highlighted the importance of facilitative management in order to ensure team morale.

Preliminary findings of the implementation of CAPA in New Zealand have shown that acceptance rates for choice appointments have increased, as have staff retention and morale. Clinicians have noted increased motivation and engagement of clients and reduced missed appointments. It is hypothesised that this increased engagement is due to the collaborative nature of the system, with a move from clinicians as 'expert with power' to 'facilitator with expertise' (Mental Health Foundation, 2009).

A benefit of the CAPA approach is that most clients who are referred to a CAMHS are seen for an appointment, even if it is just a choice appointment. This naturally leads to increased access and eliminates waitlists. The flexibility of the choice appointment, in terms of time and location, increases the likelihood of attendance. The collaborative nature of the choice appointment is intentional in order to enhance engagement.

\section{Reminders}

Some CAMHS have been utilising text reminders for appointments. These reminders are resulting in increased attendance rates, especially for first appointments. Given that most young people have a mobile phone and are comfortable communicating via text, it is seen as an effective way to remind young people about appointments.

Some clinicians use appointment cards, writing down the next appointment time at the end of each session. Again, this clarity about appointment times seems to increase the likelihood of attendance. 


\section{Cultural advisors}

Cultural advisors are a key aspect of enhancing engagement in CAMHS. Some CAMHS have dedicated cultural workers who are available exclusively for the team. Other CAMHS have access to health board cultural advisors. On-site cultural workers are often the difference between young people and their families engaging or not. Sometimes this is simply due to identifying with ethnicity, other times it is related to bridging the gap between the clinician and client and ensuring that the appropriate protocols or processes are followed in order to make the family feel more at ease. When cultural workers are not on site, health board cultural advisors can assist with providing advice regarding cultural issues or, for example, in the case of Maori, specific iwi-based knowledge that may help improve awareness and thus increase the chances of engagement.

\section{Individualised approaches}

Alongside the initiatives above, there are some approaches that can be tailored to each client. Often in practice, clinicians can get a sense of whether someone is minimising their difficulties and is embarrassed about getting help, or if they really do not require specialist services. This does take experience to distinguish between the two presentations. Often it can be factors such as cultural difference, economic disadvantage and multi-stressed families that contribute to engagement difficulties. Taking an individualised approach relies upon having resourcing within the team to depart from a standardised model, which also requires clinician availability.

When clinicians are able to understand these structural barriers, and are resourced to extend their practice, it can be very effective to offer appointments that are at home or school. This takes some of the stress out of clinic attendance for multi-stressed families. It also means that the appointment occurs in a context that is familiar to the family, where they feel comfortable and may be more likely to talk freely.

Having the time to build rapport slowly, without the pressure of completing an assessment, can also enhance engagement. Again, this relies upon clinicians having the time to do this. Extra work put into initial engagement and rapport building can have long-lasting effects, particularly for those young people and families who have a mistrust of mental health services.

Resourceful and creative clinicians use a wide variety of techniques for engaging young people. Clinicians can use what they know of the young person to engage them. The use of art and music can help young people to feel more relaxed, and can greatly aid the assessment.

The location of appointments can be changed. Clinicians sometimes have appointments with young people at school, the beach, marae, local parks or even places like McDonald's. It is important to maintain professional boundaries, so the rationale for an alternative location for appointments should be considered. It may be that a young person would struggle to physically get to the clinic, and their home is overcrowded, so an alternative location is preferable. In addition, many CAMHS clinics are hospital-based, which can be a barrier for some young people attending due to the perceived stigma of attending a mental health service on a hospital site. Alternative locations for appointments can therefore contribute to increased engagement with the young person. 
'Family' can be considered in a wider context, with the involvement of trusted others in the engagement and assessment process, such as ministers, friends and neighbours. With the consent of the family, utilising the resources of people who know the young person well can greatly assist engagement. All of these ideas involve thinking outside of the box, moving away from a clinical environment, to one where the young person feels at ease.

\section{Working with referrers}

Referrers have a very important role in enhancing engagement. Referrers can model a non-stigmatising attitude towards mental health. This greatly improves the perception of CAMHS by young people and their families, thereby directly addressing a significant barrier to engagement. It is important for referrers, including social workers, to understand mental health issues, signs of distress and when to refer to CAMHS. Enhanced knowledge about mental health issues means that social workers who work with young people outside of CAMHS are equipped to recognise adolescent mental health issues, and can make appropriate referrals. Often it is social workers who know families well, and have the opportunity to be a link between young people and CAMHS.

Alongside knowledge of mental health issues, it is also important for referrers to know about what the local CAMHS can offer, and the process for assessing young people and their families. This information can be shared with families, so they have realistic expectations about the service they will receive, and also allay fears about the process.

Referrers and CAMHS clinicians should work as partners. This involves getting to know each other. Some CAMHS have liaison programmes where certain clinicians are connected to certain schools, CYF offices or NGOs. Relationships build between clinicians and referrers, to the point where referrers know the clinicians well and feel comfortable to call to discuss potential referrals. In areas where these liaison programmes are not set up, it can be useful for referrers to call CAMHS services and talk to the duty or triage team. This builds relationships, and is a way to ensure appropriate referrals are received. Most CAMHS clinicians are willing to discuss issues with referrers who are unsure if a young person would meet criteria for CAMHS.

Referrers can collaborate with CAMHS clinicians and clients to problem-solve practical barriers, such as transport and child care. Establishing good relationships between referrers and CAMHS ensures that referrers can accurately explain the service to young people and help them understand what they should expect. Some CAMHS have liaison programmes with local schools and Child, Youth and Family offices in order to improve communication and understanding of each other's roles.

\section{Practice examples}

Social workers in CAMHS are charged with focusing on young people and their social environment. There are practice examples of ways in which social workers have identified barriers to engagement, and have made individualised plans to build engagement with young people and their families.

One such example involved identifying a parent's psychological barrier to engagement. An adolescent girl was referred to a CAMHS. The presenting issues were self harm and thoughts of suicide. The assessment highlighted the relationship difficulties that the girl 
had with her family, particularly her mother. The social worker made a plan to offer family therapy to all family members, including the mother. Several invitations were sent to the mother to attend family sessions, which were declined. The social worker then sent a letter to the mother explaining that she is important in the family system, and it would be most beneficial for the girl if the mother could also attend appointments. This was followed up by a phone call to the mother. It was discovered that the mother was under a lot of stress at work, and struggled to get time off for appointments. She also had a belief that her daughter's issues were personal, and should not involve the family. Providing information about the philosophy of family involvement in CAMHS, along with reassurance that the mother would not be blamed for her daughter's difficulties, resulted in the mother agreeing to attend appointments. The mother and social worker negotiated times for the meetings that would be less disruptive to the mother's work. As a result the family attended six months of family therapy. The girl stopped self-harming and worked with her parents towards more open communication about her needs. The family prioritised family time on the weekends, and the girl felt less isolated by building strong connections with her family.

An example of clinical work that addressed cultural barriers involved a Tongan girl and her family. She was referred by her school counsellor for depressed mood. Several appointment letters were sent to her family, but they did not attend. There was no phone line, and so the decision was made by the social worker to engage the help of a cultural worker to explain the referral and the CAMHS role to the family in-person. The social worker and cultural worker met the family at their home, and took time to explain fully the processes involved and build rapport. The cultural worker helped to de-stigmatise the process by giving explanations about the helping role. He discovered that the family had a cultural explanation for the girl's behaviour, and explained that this would be incorporated in the assessment. This first meeting focused solely on giving information about CAMHS and building rapport. The rationale was that a slower process would lead to a more robust assessment further down the track. Given that CAMHS services are usually explained by telephone, this was an unorthodox approach to informing a family about the referral and service. However, it was effective as it involved culturally-appropriate modes of engagement, including a slower approach to assessment. The girl took a long time to trust the social worker, but when she did she was able to disclose serious suicidal thoughts, including being in a suicide pact with other young people. Taking a culturally appropriate approach to engagement meant that an adolescent with high needs and serious mental health difficulties was able to receive the assistance she required.

A Chinese adolescent male was referred with anxiety symptoms. During the triage process, clinicians were finding it difficult to make contact with the parents, so they enlisted the help of an interpreter to make initial telephone contact. From there, the parents became more responsive to phone calls and conversations about their son, and agreed to attend an initial assessment. An interpreter was also present at the assessment and was able to interpret the contents of the discussion. This facilitated a process of engagement resulting in the family returning for subsequent appointments and ongoing treatment. Interpreting services continued to be used for the parents, while their son was able to complete treatment with an English-speaking clinician. 


\section{Conclusion}

Given the level of mental health issues among the New Zealand child and adolescent population, engagement is a crucial component to enhancing wellbeing and the success of recovery. Psychological, cultural and practical barriers to engaging with mental health services need to be addressed in order to ensure that services are reaching those most in need. The literature promotes the use of interventions that target all of these barriers when engaging young people and their families. Systemic interventions that are multi-layered fit well with a social work paradigm given their focus on the interaction between a person and their environment. Social workers have a role in advocating this type of approach within their service.

Enhancing engagement in CAMHS can be a challenge, however there are a number of interventions that have shown success. The initial stages of psychological interventions are critical for building rapport and a collaborative working relationship, thereby helping with engagement. This should occur in the context of working with the family, bringing family members together to work towards shared goals. Cultural interventions are also extremely significant at the outset in order for families to feel comfortable attending a mainstream service. A recent engagement initiative is the Choice and Partnership Approach. This aims to streamline services, reduce waitlists, and increase access rates for those requiring support. Alongside this standardised model, clinicians are also using individualised approaches to respond to complex situations.

The youth of New Zealand need approaches to mental health services that are growing and evolving and that are responsive to their experience of living in New Zealand. Social workers need to constantly reassess their approaches to engagement in order to meet these needs. This should occur at an individual, collegial and service-based level in order to maintain best practice and serve well some of our most vulnerable population.

\section{References}

Adolescent Health Research Group. (2008). Youth'07: The health and wellbeing of secondary school students in New Zealand. Initial findings. Auckland: The University of Auckland.

Bryan, J. (2009). Engaging clients, families, and communities as partners in mental health. Journal of Counseling and Development, 87, 507-512.

Clark, T.C., Robinson, E., Crengle, S., Herd, R., Grant, S., \& Denny, S. (2008). Te ara whakapiki taitamariki. Youth'07: The health and wellbeing survey of secondary school students in New Zealand. Results for Māori young people. Auckland: The University of Auckland.

Fergusson, D., Poulton, R., Horwood, J., Miln, B., \& Swain-Campbell, N. (2003). Comorbidity and coincidence in the Christchurch and Dunedin longitudinal studies. Wellington: Ministry of Social Development/Department of Labour/The Treasury.

Gulliver, A., Griffiths, K.M., \& Christensen, H. (2010). Perceived barriers and facilitators to mental health help-seeking in young people: A systematic review. BMC Psychiatry, 10, 113-131.

Guy, R., Hocking, J., Wand, H., Stott, S., Ali, H., \& Kaldor, J. (2012). How effective are Short Message Service reminders at increasing clinic attendance? A meta-analysis and systematic review. Health Services Research, 47(2), 614-632.

Ingoldsby, E.M. (2010). Review of interventions to improve family engagement and retention in parent and child mental health programs. Journal of Child and Family Studies, 19(5), 629-645.

Kim, H., Munson, M.R., \& McKay, M.M. (2012). Engagement in mental health treatment among adolescents and young adults: A systematic review. Child and Adolescent Social Work Journal, 29, 241-266.

McKay, M.M., \& Bannon, W.M. (2004). Engaging families in child mental health services. Child and Adolescent Psychiatric Clinics of North America, 13, 905-921.

Mental Health Commission. (2012). Blueprint II: How things need to be. Wellington: Mental Health Commission.

Mental Health Foundation. (2009). Evaluation of the choice and partnership approach in child and adolescent mental health services in England. England: National CAMHS Support Service. 
Ministry of Health. (2007). Te raukura mental health and alcohol and other drugs: Improving outcomes for children and youth. Wellington: Ministry of Health.

Ministry of Social Development. (2010). The social report. Wellington: Ministry of Social Development.

Pescosolido, B.A., Brooks Gardner, C., \& Lubell, K.M. (1998). How people get into mental health services: Stories of choice, coercion and 'muddling through' from 'first timers'. Social Science Medicine, 46(2), $275-286$.

Rickwood, D., Deane, F.P., Wilson, C.J., \& Ciarrochi, J. (2005). Young people's health-seeking for mental health problems. Australian e-Journal for the Advancement of Mental Health, 4(3) Supplement from www.auseinet.com/ journal/vol4iss3suppl/ rickwood.pdf.

Robotham, D., James, K., \& Cyhlarova, E. (2010). Managing demand and capacity within child and adolescent mental health services: An evaluation of the choice and partnership approach. Mental Health Review Journal, 15(3), 22-30.

The Werry Centre. (2011). 2010 stocktake of infant, child and adolescent mental health and alcohol and other drug services in New Zealand. Auckland: The Werry Centre for Child \& Adolescent Mental Health Workforce Development, The University of Auckland.

Wille, A. (2006). Whakamārama te huarahi - to light the pathways: A strategic framework for child and adolescent mental health workforce development 2006-2016. Auckland: The Werry Centre for Child and Adolescent Mental Health Workforce Development.

York, A., \& Kingsbury, S. (2009). The choice and partnership approach: A guide to CAPA. Bournemouth: Caric Press. 